\title{
HISTOGRAM EXPANSION-A TECHNIQUE OF HISTOGRAM EQULIZATION
}

\author{
Jasdeep Kaur ${ }^{1}$, Nancy $^{2}$, Nishu $^{3}$, Ramneet Kaur ${ }^{4}$ \\ ${ }^{1,2,3,4}$ M.Tech, Guru Nanak Dev Engg College, Ludhiana
}

\begin{abstract}
In this paper I have described histogram expansion. Histogram expansion is a technique of histogram equalization. In this I have described three different techniques of expansion namely dynamic range expansion, linear contrast expansion and symmetric range expansion. Each of these has their specific uses and advantages. For colored images linear contrast expansion is used. These all methods help in easy study of histograms and helps in image enhancement.
\end{abstract}

Index Terms: Histogram expansion, Dynamic range expansion, Linear contrast expansion, Symmetric range expansion $* * *$

\section{INTRODUCTION}

\section{HISTOGRAM}

Histogram can be defined as the graphical distribution of pixels over the tonal range or luminous intensity.

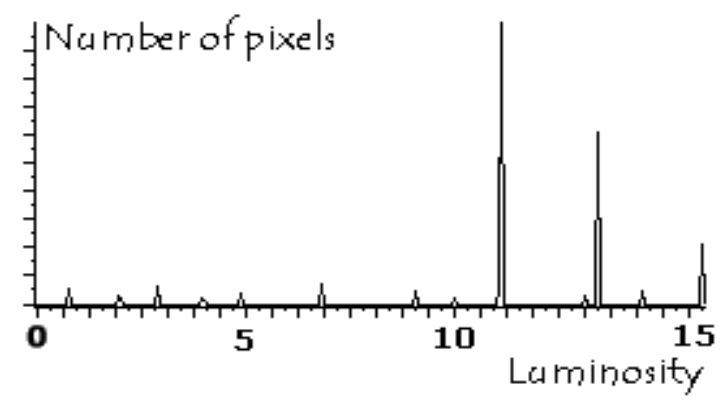

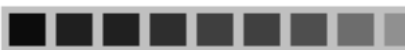

Figure 1

We can do modification of histogram in order to correct the contrast of under-exposed and over-exposed images. Histograms are also used to study distribution of various components of the images. Various histogram processing are histogram equalization and histogram matching. In this section histogram expansion-a technique of histogram equalization have been described.[1]

\section{HISTOGRAM EQUALIZATION}

Histogram equalization can be described as method in which histogram is modified by spreading the gray level areas.this can be shown as;

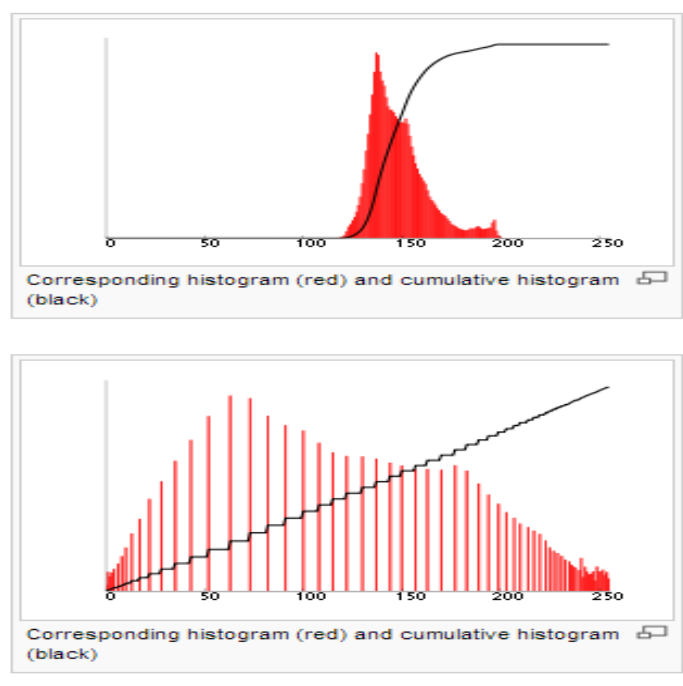

Figure 2

Let us consider an input histogram $h_{i}$ and that produces a modified histogram $\mathrm{h}$ by histogram expansion that is close to any uniformly distributed histogram $\mathrm{u}$, then histogram equalization can be described as :

$$
\min \left\|\mathrm{h}-\mathrm{h}_{\mathrm{i}}\right\|+\lambda\|\mathrm{h}-\mathrm{u}\|
$$

\section{ADJUSTABLE HISTOGRAM EXPANSION}

For this we have

$$
h^{\prime}=\frac{h_{i}+\lambda u}{1+\curlywedge}=\left(\frac{1}{1+\lambda}\right) h_{i}+\left(\frac{\lambda}{1+\lambda}\right) u
$$

By using this equation we show enhancement of image. 


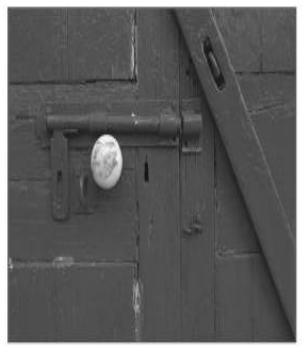

(a)

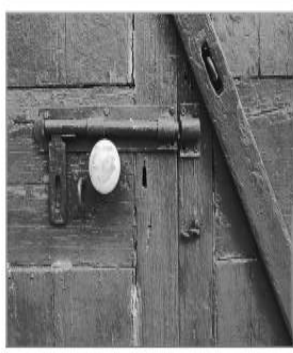

Fig.3 Modticed histogram equalization results using 2 f for image Door. (a) Oniginal image, (b) cahanned image using 12 with $\lambda=0$, (c) enhanced image using 2) with $\lambda=1$,(d) enhanced inage using (6) with $\lambda=2$.

Figure 3

There are various methods present for histogram equalization. Few of these are histogram expansion which is described in this paper. others are Dualistic sub-image histogram equalization(DSIHE), by modifying 'cumulationfunction', gray level grouping(GLG),etc.all these techniques are used for image enhancement purposes.[2][3]

\section{HISTOGRAM EXPANSION}

This is denoted by HE. It is explained as remap of a sub-range intensity of an input image to full range in an output image. Input range is $\mathrm{x}$ [xmin, $\mathrm{xmax}]$ and output range to which it is stretched is $y[0,255]$. This mapping can be described by using the following linear mapping function:

$$
\mathrm{y}=\frac{\mathrm{x}-\mathrm{xmin}}{\mathrm{xmax}-\mathrm{xmin}} \mathrm{ymax}
$$

histogram expansion can be shown in figure 4 .

\section{MODIFIED HISTOGRAM EXPANSION}

Provides greater enhancement in the cases where histograms have narrow peak and tails to end of each side of peak. In this we have new lower and upper bounds described as:

$$
\mathrm{xmin}^{\prime}: \frac{(100-\mathrm{P})}{2}=\frac{100}{\mathrm{~T}} \sum_{\mathrm{x}=\mathrm{xmin}}^{\mathrm{xmin}}{ }^{\prime} \mathrm{h}(\mathrm{x})
$$

Where $\mathrm{T}=\sum_{\mathrm{xmin}}^{\mathrm{xmax}} \mathrm{h}(\mathrm{x})$

$$
\operatorname{xmax}^{\prime}: \frac{(100-\mathrm{P})}{2}=\frac{100}{\mathrm{~T}} \sum_{\mathrm{x}=\mathrm{xmax}}^{\mathrm{xmax}} \mathrm{x}^{\prime} \mathrm{h}(\mathrm{x})(5)
$$

Where $\mathrm{P}$ is user selected cut-off percentage and it ranges from $98 \%$ down to $85 \%$.

The mid $\mathrm{P} \%$ of intensities are stretched to full range and tails that comprise of (100-P)/2\%each are compressed.[4]

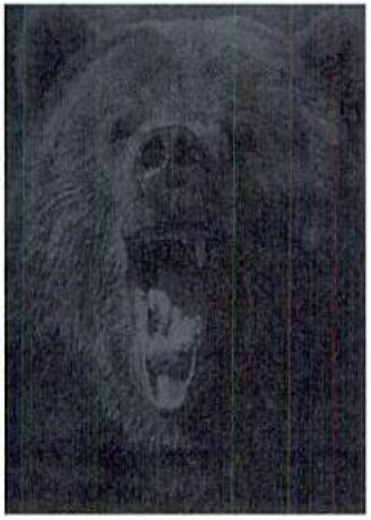

(a)

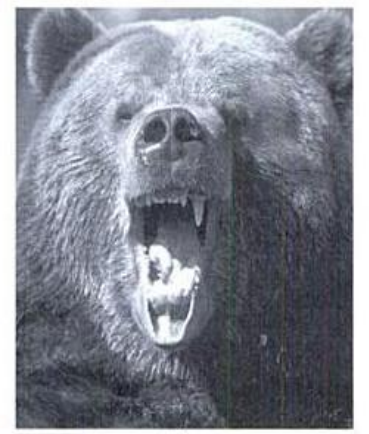

(c)

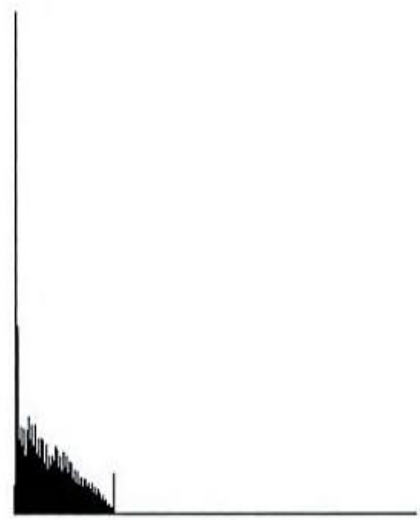

(b)

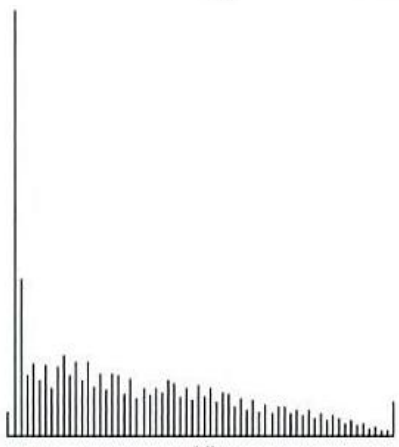

(d)
Figure 4 Pictorial Example. Illustration of contrast enhancement by histogram expan. sion: (a) original image with poor contrast; (b) histogram of (a), the narrow range of occupied histogram bins indicating poor contrast, the concentration of intensities at the high end indicating dark image; (c) result of enhancing contrast in (a); (d) histogram of (c). (This product/publication includes images from Corel Stock Photo Library, which are protected by the copyright laws of the U.S., Canada, and elsewhere. Used under license.)

Figure 4

\section{DYNAMIC RANGE EXPANSION:}

Dynamic range expansion can be explained as expansion of unused range. This can be on both the sides i.e. lower bound and upper bound here pixel values remain same. This is mainly done so that visualizing the image can be relived from stress and fatigue i.e. into range that suits normal eye.

This is also called as normalization. There can be two kindslinear and nonlinear normalization.

We can explain this by taking initial range as I: $\{$ Min ,Max $\}$ And new range as IN: $\{$ newMin , newMax then linear normalization is as shown:

$$
\mathrm{I}_{\mathrm{N}}=(\mathrm{I}-\mathrm{Min}) \frac{(\text { newMax }- \text { newMin })}{(\operatorname{Max}-\text { Min })}+\text { newMin }
$$




$$
\left.\mathrm{I}_{\mathrm{N}=(\text { newMAx }}-\text { newMin }\right) \frac{1}{1+\mathrm{e}-\frac{\mathrm{I}-\beta}{\alpha}}+\text { newMin }
$$

Where $\alpha$ is width of input intensity range and $\beta$ is intensity around which range is centered.

Advantage of dynamic range expansion is that it can preserve the losses that occur due to contrast gains or clippings as in case of shadows, or in photographs that have back lit background (eg direct sunlight, light snow).

Upper and Lower dynamic range expansion is shown in fig 5.[5][6]
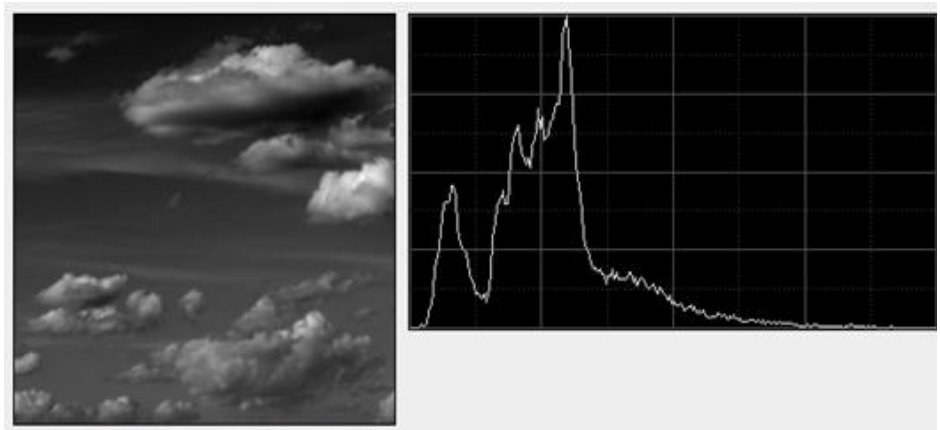

The original image and its histogram.
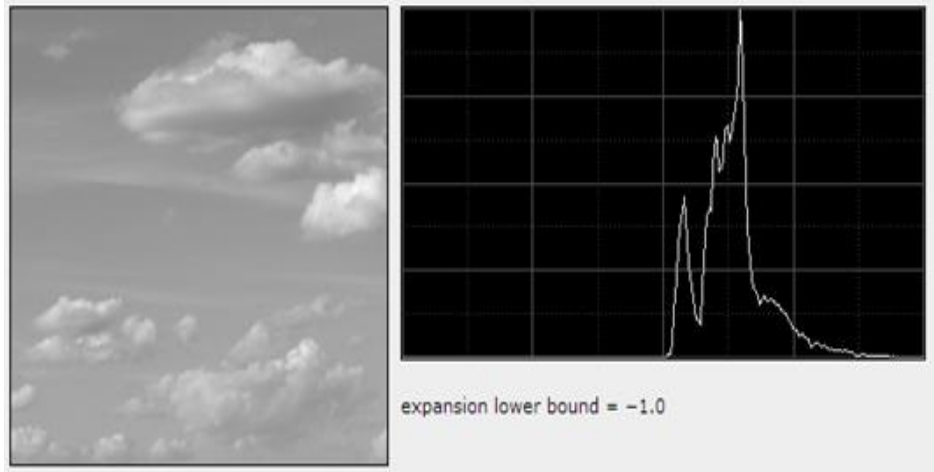

expansion lower bound $=-1.0$
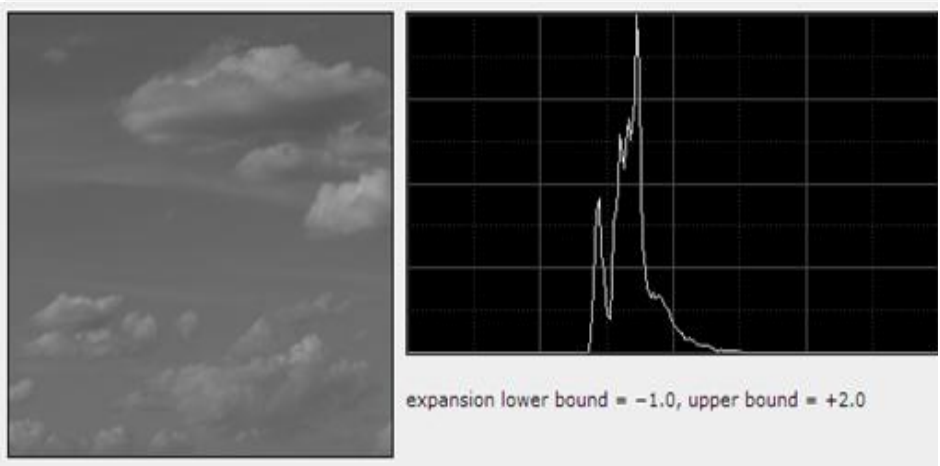

expansion lower bound $=-1.0$, upper bound $=+2.0$

\section{LINEAR CONTRAST EXPANSION}

This method is used to solve brightness saturation problem. Visibility is improved by stretching the contrast this is done by assigning the new values to the pixels. The pixels to the darkest side are talen as black and to lightest side are taken aswhite. The pixels lying between light and dark are changed the pixel values to improve the contrast.

This is also applied in color images. This is done by increasing the intensity, lightness while leaving the color information same. We can also produce color shifts in image by expanding the RGB values fully individually, but the colors should be few or only single color. Expansion may make it possible to compare images acquired with slightly different brightness ranges by adjusting them all to same expanded contrast scale. This only works if brightest and darkest images are present in all of image.

New gray level that is obtained after contrast stretching can be mathematically explained as:

(8) $\mathrm{Y}=\left[\frac{(\mathrm{x}-\mathrm{xmin})}{(\mathrm{xmax}-\mathrm{xmin})}\right](y \max -y \min )+y \min$

Where (xmin, $x \max$ ) is range of original image and (ymin,ymax) are range of final image.

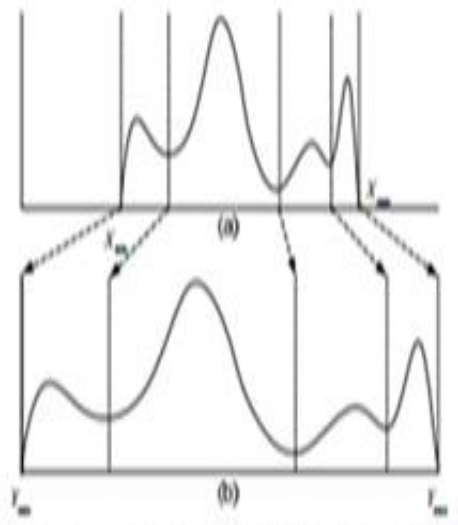

Foure 6 linear contast expansion (a) Original histogran (b) New histogram.

Figure 6

\section{APPLICATIONS OF CONTRAST STRETCHING}

Its use in microscopic images can be shown in following figures.

Figure 5 


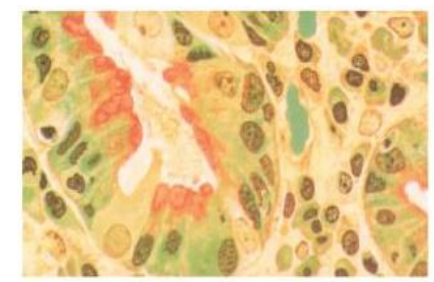

(a)

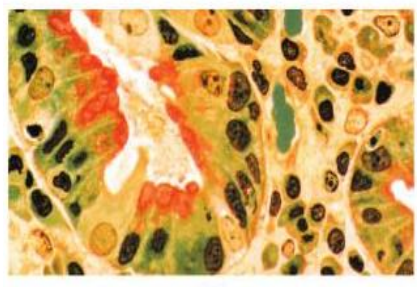

Figure 7

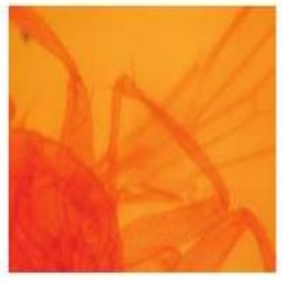

(a)

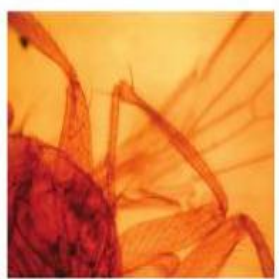

(c)

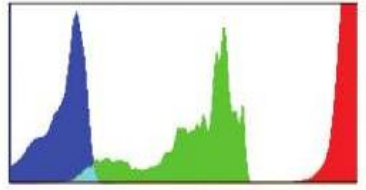

(b)

Figure 8 Ligbt microscope image of an insect (a) with bistograms of the red, green, and blue values (b). Expanding the intensity wbile leaving bue and saturation unchanged $(c)$ expands the visible con. trast witbout altering the colors. Expanding the $R G B$ values individually to full range produces color shifts in the image (d) because the propor. tions of red, green and blue are changed. differ by 1 bit. 2 and 3 are1-buddies of each other,4,5,6,7, are 2 buddies of each other. In this expansion is done by moving the pixel value to empty neighbor n-buddy.Embedding process can be mathematically written as:

$$
\mathrm{C}_{\mathrm{W}}=\left[\mathrm{C} / 2^{\mathrm{n}}\right] \cdot 2^{\mathrm{n}}+\mathrm{W}
$$

This is shown in following figures 9 and 10 .
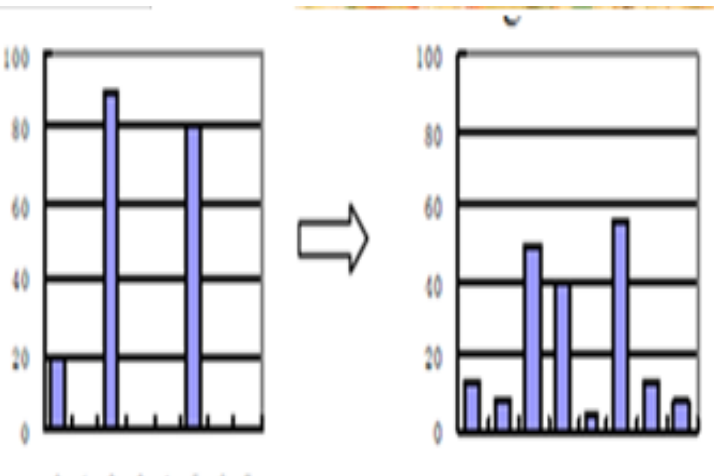

01218561

01251561

Fig g, Histogram modification for embedding data

Figure 9

\section{ADVANTAGES}

(1)The algorithm is simple one.

(2)There is no need to evacuate all the bins for embedding purpose because pivot bins can be shifted on both sides (3)Here fewer watermarks bits need histogram shifting

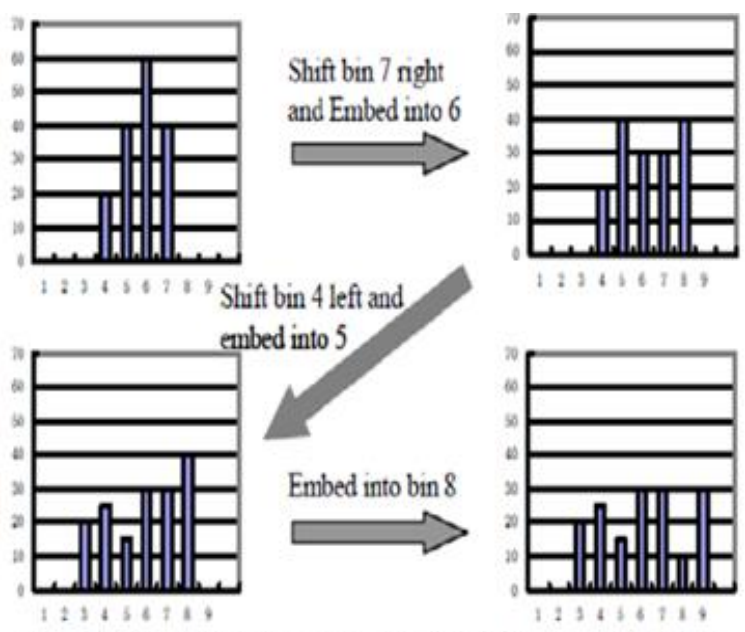

Fig10Symmetrical histogram expansion for data embedding

Figure 10

(4)This results in enhanced images shown in figure.[8] 


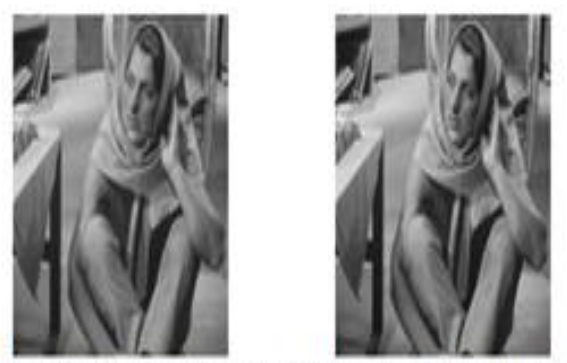

Fig11The original image (Left) and the watermarked image with 16298 byte hidden data and $34.04 d B$ PSNR (Rightit)

\section{Figure 11}

\section{CONCLUSION}

Histogram expansion leads to enhancement of image .This is mainly used when histogram contain one very tall spikes and it becomes difficult to interpretthe rest of information. Various methods like symmetric histogram expansion can be used for study of these histograms. The images with shadows can be clearly observed using dynamic range expansion. Contrast expansion can be used to solved brightness issues.Each technique of expansion have its own uses. Further many more algorithms can be explained for this technique of histogram expansion. Also we can use various methods of histogram equalization for improving contrast of images.

\section{REFERENCES}

[1]“Histogram"'http://en.kioskea.net/contents/video/traitimg.p hp3

[2] TarikArici, SalihDikbas, YucelAltunbasak "A Histogram Modification Framework and Its

Application for Image Contrast Enhancement" IEEE,2009

[3]"Histogram Equlization" Wikipedia.org

[4]Lawrence

o.

Gorman,Michaelj.Sammon,MichaelSeul,Practical algorithms for image analysis,secondedition,Cambridge

[5]"HistogramTransformation",http://pixinsight.com/doc/tools /HistogramTransformation/HistogramTransformation.html

[6]"normalization(image processing)",Wikipedia.com

[7]John C. Russ, The Image processing handbook ,sixth edition,Taylor and Fransis group

[8] L. Yanga ,P. Haoa, C.Zhanga, "PROGRESSIVE REVERSIBLE DATA HIDING BY SYMMETRICAL HISTOGRAM EXPANSION WITH PIECEWISE-LINEAR HAAR TRANSFORM" University of London,2007 